\title{
REORDENAMIENTO VEHICULAR Y CONTAMINACIÓN AMBIENTAL POR MATERIAL PARTICULADO (2,5 Y 10), DIÓXIDO DE AZUFRE Y DIÓXIDO DE NITRÓGENO EN LIMA METROPOLITANA, PERÚ
}

\author{
Vilma Tapia1,a, Luz Carbaja|2,b, Vanessa Vásquez ${ }^{1, \mathrm{c}}$, Rocío Espinoza $^{3, \mathrm{~d}}$, Cinthya Vásquez-Velásquez ${ }^{1, \mathrm{e}}$, \\ Kyle Steenland ${ }^{4, f}$, Gustavo F. Gonzales ${ }^{1, g}$
}

\begin{abstract}
RESUMEN
Objetivos. Evaluar el cambio en los niveles ambientales de material particulado (2,5 y 10) $\left(\mathrm{PM}_{25}\right.$ y $\left.\mathrm{PM}_{10}\right)$, dióxido de azufre $\left(\mathrm{SO}_{2}\right)$ y dióxido de nitrógeno $\left(\mathrm{NO}_{2}\right)$, posterior al reordenamiento vehicular en la avenida Abancay, entre 20132015 respecto a 2007-2009, comparando con otras dos avenidas sin implementación de un reordenamiento, la avenida Guillermo de La Fuente en el distrito de Comas y la avenida César Vallejo en el distrito de El Agustino. Materiales $\boldsymbol{y}$ métodos. Los datos ambientales fueron colectados por tres monitores de Dirección General de Salud Ambiental e Inocuidad Alimentaria del Ministerio de Salud como parte de la red de Vigilancia de la calidad del aire de la ciudad de Lima. El coeficiente beta de la regresión lineal permitió evaluar el cambio en la concentración de cada contaminante. Resultados. El reordenamiento de la Av. Abancay redujo $62 \%$ los niveles de $\mathrm{PM}_{2,5} ; 55 \%$ los niveles de $\mathrm{PM}_{10} ; 65 \%$ los niveles $\mathrm{NO}_{2}$; y $82 \%$ los de $\mathrm{SO}_{2}$. En las otras dos avenidas evaluadas no se observó disminución. Una reducción significativa de $\mathrm{PM}_{25}$ ( $\beta$ : $-53,11 \mu \mathrm{g} / \mathrm{m}^{3}$; IC 95\%: -63,92 a -42,30), $\mathrm{PM}_{10}\left(\beta:-47,95 \mu \mathrm{g} / \mathrm{m}^{3}\right.$; IC 95\%: $-62,61$ a $\left.-33,37\right), \mathrm{NO}_{2}$ $\left(\beta:-41,71 \mu \mathrm{g} / \mathrm{m}^{3}\right.$, IC 95\%: $-48,18$ a $\left.-35,23\right)$ y SO 2 ( $\beta$ : $-28,59 \mu \mathrm{g} / \mathrm{m}^{3}$; IC 95\%: $-35,23$ a $\left.-21,95\right)$ fueron observados en la $A v$. Abancay, respecto a las otras dos avenidas, posterior al reordenamiento vehicular. Conclusiones. El reordenamiento vehicular puede contribuir con la disminución continua de diversos contaminantes ambientales. La extensión de esta medida podría mejoraría la calidad de aire y estado de salud de la población residente en la ciudad de Lima.
\end{abstract}

Palabras clave: Salud ambiental; Contaminación del aire; Contaminantes atmosféricos; Material particulado; Perú (Fuente: DeCS BIREME).

\section{TRAFFIC REGULATION AND ENVIRONMENTAL POLLUTION BY PARTICULATE MATERIAL (2.5 AND 10), SULFUR DIOXIDE, AND NITROGEN DIOXIDE IN METROPOLITAN LIMA, PERU}

\begin{abstract}
Objective. To assess the change in environmental levels of PM2.5, PM10, SO2, and NO2, after the application of a traffic regulation plan in Abancay Avenue between the years $2013-2015$ vs. to $2007-2009$, and comparing it against two other avenues, in which no traffic regulation was implemented: Guillermo de La Fuente Avenue in the district of Comas and Cesar Vallejo Avenue in El Agustino. Materials and Methods. Environmental data were collected by three air monitors from the Ministry of Health's Environmental Health and Food Safety General Directorate (Dirección General de Salud Ambiental e Inocuidad Alimentaria) that are part of the Air Quality Surveillance network of the city of Lima. The beta coefficient of the linear regression allowed to assess change in the concentration of each pollutant. Results. The traffic regulation plan of Abancay Av. reduced the levels of PM2.5 by $62 \% ; 55 \%$ for PM10 levels; $65 \%$ for the NO2 levels, and $82 \%$ for the SO2 levels. In the other two avenues assess, this reduction was not observed. A significant reduction in PM2.5 ( $\beta$ : $-53.11 \mu \mathrm{g} / \mathrm{m3}$; 95\% Cl: -63.92 to -42.30$)$, PM10 ( $\beta$ : $-47.99 \mu \mathrm{g} / \mathrm{m3} ; 95 \% \mathrm{Cl}:-62.61$ to -33.37$)$, NO2 ( $\beta$ : $-41.71 \mu \mathrm{g} /$ m3; $95 \%$ Cl: -48.18 to -35.23 ), and SO2 ( $\beta$ : $-28.59 \mu \mathrm{g} / \mathrm{m3} ; 95 \% \mathrm{Cl}:-35.23$ to -21.95$)$ was observed in Abancay Av., with respect to the other two avenues, after the traffic regulation plan was implemented. Conclusions. Traffic regulation can contribute to the continuous reduction of different air pollutants. The expansion of this measure could improve air quality and the health status of the residents of Lima.
\end{abstract}

Keywords: Environmental health; Air pollution; Air pollutants; Particulate matter; Peru (Source: MeSH NLM).

\footnotetext{
Departamento de Ciencias Biológicas y Fisiológicas, Facultad de Ciencias y Filosofía, Universidad Peruana Cayetano Heredia. Lima, Perú.

Departamento de Estadística, Demografía, Humanidades y Ciencias Sociales, Universidad Peruana Cayetano Heredia. Lima, Perú.

Dirección General de Salud Ambiental e Inocuidad Alimentaria (DIGESA). Ministerio de Salud. Lima, Perú.

Department of Environmental Health, Rollins School of Public Health, Emory University. Atlanta, GA, USA.

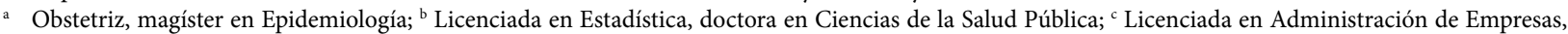
magíster en Economía y Mercados Emergentes; ${ }^{\mathrm{d}}$ Médico Cirujano; ${ }^{\mathrm{e}}$ Licenciado en Biología; ${ }^{\mathrm{f}} \mathrm{PhD}$ en Epidemiología; ${ }^{\mathrm{g}}$ Médico Cirujano, doctor en Ciencias y doctor en Medicina.

Recibido: 15/11/2017 Aprobado: 18/04/2018 En línea: 03/07/2018
}

Citar como: Tapia V, Carbajal L, Vásquez V, Espinoza R, Vásquez-Velásquez C, Steenland K, et al. Reordenamiento vehicular y contaminación ambiental por material particulado (2,5 y 10), dióxido de azufre y dióxido de nitrógeno en Lima Metropolitana, Perú. Rev Peru Med Exp Salud Publica. 2018;35(2):190-7. doi: 10.17843/ rpmesp.2018.352.3250. 


\section{INTRODUCCIÓN}

La Organización Mundial de la Salud (OMS) estima que en 2012 la contaminación del aire en el mundo generó tres millones de muertes prematuras al año ${ }^{(1)}$, siendo su efecto mayor en las megaciudades ${ }^{(2)}$. En Lima, la capital del Perú con más de diez millones de habitantes, el $80 \%$ de la contaminación del aire proviene del parque automotor ${ }^{(3)}, y$ en los últimos 20 años la cantidad de vehículos motorizados se ha incrementado notoriamente ${ }^{(4)}$.

Según la Encuesta de Recolección de Información Básica de Transporte Urbano en el área metropolitana de Lima y Callao, realizada el 2012 por la Agencia de Cooperación Internacional del Japón (JICA) ${ }^{(5)}$, el número de viajes que se realizan al día es de 22,3 millones, de ellos 16,9 millones corresponden a vehículos motorizados, y se estima que las cifras aumenten junto con las emisiones de gases vehiculares.

Las autoridades tratando de mitigar este problema implementaron estrategias orientadas a reducir las emisiones. La eliminación del plomo de la gasolina determinó una drástica reducción de la contaminación de plomo ${ }^{(6-8)}$. Igualmente, se estableció la reducción del contenido de azufre en el diésel (9), la obligatoriedad de las revisiones técnicas vehiculares ${ }^{(10)}$, y una disminución de la antigüedad permisible para la circulación vehicular ${ }^{(11)}$. A pesar de ello, la OMS en 2014 reportó que Lima es la ciudad con mayor contaminación en América Latina ${ }^{(12)}$, lo que hace pensar que estas estrategias no fueron suficientes para reducir la contaminación o que las causas procedan de otras fuentes.

Asimismo, el Congreso de la República e instituciones gubernamentales peruanas han realizado estrategias para aminorar los problemas de contaminación del aire. Mediante los incisos 7.1 a 7.7 del artículo $161^{\circ}$ de la Ley Orgánica de Municipalidades (Ley 27972), se acordó planificar, regular y gestionar el transporte público, privado, motorizado menor y no motorizado; así como también, se estableció un monitoreo de los sistemas de señalización, terminales terrestres, tránsito peatonal, entre otros ${ }^{(13)}$.

Una de las estrategias iniciales fue el plan de ordenamiento del tráfico vehicular, como parte del Comité de Gestión de Iniciativa de Aire Limpio para Lima y Callao (CGIALLC), que consistió en ordenar la circulación de vehículos en función de la estructura urbana, para mejorar la fluidez del tránsito vehicular ${ }^{(14)}$. El plan de ordenamiento de las principales avenidas inició entre noviembre y diciembre de 2011 con las avenidas Túpac Amaru y Abancay, respectivamente, y años posteriores en las avenidas Tacna, Canadá, y Javier Prado, entre otras. Otros proyectos ejecutados para reducir la congestión vehicular fueron Vía Parque Rímac, Túnel Santa Rosa, Remodelación de la Av. Canadá y obras de mantenimiento vial $(150 \mathrm{~km})^{(15)}$.

\section{MENSAJES CLAVE}

Motivación para realizar el estudio. La transformación en el sistema de transporte es tema de gran interés para los gobiernos, debido a que el crecimiento de las ciudades debe ir de la mano con un ordenamiento vial para que el avance sea sostenible.

Principales hallazgos. Se evidencia aspectos positivos del reordenamiento en la avenida Abancay en la ciudad de Lima, que se asocia con una disminución de contaminantes ambientales.

Implicancias. Los aspectos positivos del reordenamiento vial en la contaminación ambiental pueden servir de base para continuar con diferentes políticas e intervenciones para beneficio de toda la población.

En el marco del plan de actividades 2011 del CGIALLC ${ }^{(16)}$, la Municipalidad Metropolitana de Lima implementó un plan de ordenamiento de la Av. Abancay en la que se aplicaron medidas administrativas de tránsito y transporte con el propósito de disminuir la congestión vehicular y la contaminación ambiental.

En este contexto, el objetivo del presente estudio fue analizar los niveles de concentración de los contaminantes ambientales como material particulado $2,5\left(\mathrm{PM}_{2.5}\right)$, material particulado $10\left(\mathrm{PM}_{10}\right)$, dióxido de azufre $\left(\mathrm{SO}_{2}\right)$ y dióxido de nitrógeno $\left(\mathrm{NO}_{2}\right)$ antes y después del reordenamiento vehicular de la Av. Abancay y comparar estos resultados con dos avenidas sin dicha intervención.

\section{MATERIALES Y MÉTODOS}

\section{DISEÑO DEL ESTUDIO}

Estudio ecológico que evaluó las concentraciones promedio de contaminantes ambientales, empleados como parámetros de calidad del aire, en tres zonas de Lima Metropolitana: Lima Centro, Este y Norte. La zona Lima Centro se encuentra bajo un proceso de ordenamiento vehicular desde diciembre de 2011. Las zonas de estudio fueron escogidas por la presencia de monitores ambientales con datos suficientes y cercanos a la Av. Abancay, Av. Guillermo de La Fuente, y Av. César Vallejo.

Los datos de las mediciones ambientales fueron proporcionados por la Dirección General de Salud Ambiental e Inocuidad Alimentaria (DIGESA) para el periodo del estudio comprendido entre 2007 y 2015.

\section{ÁMBITO DEL ESTUDIO}

El Cercado de Lima (Lima Centro) alberga una población de 282800 habitantes ${ }^{(17)}$, es recorrida por la Av. Abancay que presenta gran afluencia vehicular en sus once cuadras de distancia. En su trayecto se ubican oficinas 
gubernamentales, depósitos, tiendas, etc.; siendo una de las vías de mayor contaminación ambiental y sonora de la urbe. Según un estudio realizado por el Ministerio de Transportes y Comunicaciones (MTC) en $2009{ }^{(18)}$, midió el flujo vehicular de la Av. Abancay a la altura del puente Ricardo Palma, entre las 07:00 y 11:00 a. m., observando 16485 vehículos circulando y para el 2011, el flujo vehicular se redujo un $25 \%$.

Comas (Lima Norte) es un distrito emergente de la migración interna en los años 60 con una población de 541200 habitantes (17). La Av. Guillermo de La Fuente es de regular afluencia vehicular, en sus 12 cuadras de recorrido existen establecimientos comerciales, centros de salud y colegios. En esta zona se encuentran avenidas de gran circulación vehicular como la Av. Universitaria y la Av. Túpac Amaru. El flujo vehicular en la Av. Universitaria a la altura de la Av. Belaúnde fue de 6229 vehículos según el estudio del MTC ${ }^{(18)}$.

Por su parte, el distrito de El Agustino (Lima Este) con 196900 habitantes ${ }^{(17)}$, incluye dentro de su jurisdicción a la Av. César Vallejo. En sus 14 cuadras de recorrido se localizan tiendas, el hospital Hipólito Unanue, colegios, entre otros. En esta zona se encuentra un tramo de la carretera Panamericana conocida como vía de Evitamiento (gran flujo vehicular). El cruce con la vía de Evitamiento se encuentra aproximadamente a 500 metros del monitor ambiental. En 2009, el flujo vehicular por esta vía a la altura de Puente Huáscar (cruce con Av. César Vallejo) era de 6229 vehículos en la mañana ${ }^{(18)}$ y en el 2011, el flujo vehicular se redujo un $7 \%$.

\section{MEDICIONES AMBIENTALES}

El Ministerio de Salud, a través de la DIGESA cuenta en Lima Metropolitana con cinco estaciones fijas con equipos activos y automáticos, y una estación meteorológica. Se incluyeron en el estudio los registros de tres de ellos:

a. Estación CONACO/Congreso de la República: ubicada en el Cercado de Lima, en la segunda cuadra de la Av. Abancay. Inicialmente se localizó en el segundo piso de las instalaciones de la Corporación Nacional de Comercio (CONACO) y en el 2010 fue reubicado frente al edificio CONACO, en el parque Bolívar. Se registran una vez por semana las concentraciones de $\mathrm{PM}_{10}, \mathrm{PM}_{2,5}, \mathrm{NO}_{2}$ y $\mathrm{SO}_{2}$ desde el 2001.

b. Estación Santa Luzmila: localizada dentro del Centro de Salud Santa Luzmila en el distrito de Comas, al norte de la ciudad de Lima, en la cuadra 3 de la Av. Guillermo de La Fuente. Se registran las concentraciones de $\mathrm{PM}_{10}, \mathrm{PM}_{2,5}, \mathrm{NO}_{2}$ y $\mathrm{SO}_{2}$, una vez por semana desde el 2007.

c. Estación Hipólito Unanue: instalada dentro del Hospital Nacional Hipólito Unanue en el distrito de El Agustino, al este de la ciudad de Lima, en la cuadra 13 de la Av. César Vallejo. Se registran las concentraciones de $\mathrm{PM}_{10}, \mathrm{PM}_{2,5}, \mathrm{NO}_{2}$ y $\mathrm{SO}_{2}$, una vez por semana desde el 2007.

Para el estudio se analizaron los niveles de concentración de los contaminantes ambientales por periodo y los cambios registrados post aplicación de la norma (periodo 2013-2015) de $\mathrm{PM}_{10}, \mathrm{PM}_{2,5}, \mathrm{NO}_{2}$ y $\mathrm{SO}_{2}$ en las avenidas donde se ubican los monitores respectivos.

Los niveles referenciales se basan en los Estándares de Calidad Ambiental Nacional (ECA) (Resolución Ministerial No. 112-2015-MINAM); referente de 24 horas; $\mathrm{PM}_{2,5}: 25$ $\mu \mathrm{g} / \mathrm{m}^{3}, \mathrm{PM}_{10}: 150 \mu \mathrm{g} / \mathrm{m}^{3}, \mathrm{NO}_{2}: 40 \mu \mathrm{g} / \mathrm{m}^{3}$ y SO$: 20 \mu \mathrm{g} / \mathrm{m}^{3}$. Referente anual $\mathrm{PM}_{2,5}: 15 \mu \mathrm{g} / \mathrm{m}^{3}, \mathrm{PM}_{10}: 50 \mu \mathrm{g} / \mathrm{m}^{3}, \mathrm{NO}_{2}: 100$ $\mu \mathrm{g} / \mathrm{m}^{3}$ y $\mathrm{SO}_{2}: 20 \mu \mathrm{g} / \mathrm{m}^{3}$ y en el Valor Guía de la OMS ${ }^{(19-21)}$.

\section{IMPLEMENTACIÓN DEL REORDENAMIENTO VEHICULAR}

El reordenamiento vehicular de la Av. Abancay fue ejecutado en diciembre de 2011 , considerando un periodo de ventana de aproximadamente dos años, se ha determinado dos periodos: entre 2007 al 2009 «pre-reordenamiento vehicular» y entre 2013 al 2015 «post-reordenamiento vehicular». Como comparación se han evaluado los cambios (post-pre) de contaminantes ambientales en las Av. Guillermo de la Fuente y Av. César Vallejo.

Las medidas aplicadas incluyeron las 11 cuadras de la Av. Abancay que van desde la Av. Miguel Grau y el Puente Ricardo Palma. El reordenamiento incluyó tener solo tres paraderos para el transporte público, duplicar la velocidad de recorrido de los vehículos de transporte público de 10 $\mathrm{km} / \mathrm{h}$ a $20 \mathrm{~km} / \mathrm{h}$ y de los particulares de $20 \mathrm{~km} / \mathrm{h}$ a $40 \mathrm{~km} / \mathrm{h}$. Los buses tienen 15 segundos como máximo para recoger pasajeros, y se prohibió el estacionamiento de taxis en toda la avenida, así como, la prohibición de girar a la izquierda. Además, se instaló señalización de tránsito, semáforos sincronizados, vigilancia municipal y policial para el cumplimiento de las normas, junto con la prohibición del estacionamiento en la vía pública, y uso de carril derecho exclusivo para el transporte público.

\section{VARIABLES DE ESTUDIO}

Los contaminantes ambientales $\left(\mathrm{PM}_{2,5}, \mathrm{PM}_{10}, \mathrm{NO}_{2}\right.$ y $\left.\mathrm{SO}_{2}\right)$ fueron incluidos al estudio como variables continuas $\left(\mu \mathrm{g} / \mathrm{m}^{3}\right)$. La variable periodo fue definida como tiempo pre (2007-2009) y post (2013-2105) reordenamiento vehicular en la Av. Abancay. La variable zona es definida como el lugar donde se ubica el monitor de la estación. Se denominó $A b$ al monitor de la Av. Abancay; $S L$ al monitor de la Av. Guillermo de la Fuente, y $A G$ al monitor de la Av. Cesar Vallejo. La variable fecha fue el día de registro 
del contaminante ambiental. Finalmente, se incluyó una variable indicadora para la intervención $(0$, sin intervención; y 1 , con intervención).

\section{ANÁLISIS ESTADÍSTICO}

Se analizaron los cambios en las concentraciones de los cuatro contaminantes usando el paquete estadístico Stata v14.0 (Stata Corporation, College Station, Texas, USA). Las concentraciones de $\mathrm{PM}_{10}, \mathrm{PM}_{2,5}, \mathrm{NO}_{2}$ y $\mathrm{SO}_{2}$ fueron expresados en medias y desviación estándar. La prueba $\mathrm{t}$ de Student fue usada para comparar promedios de los contaminantes antes y después del reordenamiento vehicular.

Para evitar considerar la autocorrelación temporal de los datos se procedió a realizar una regresión lineal donde la variable dependiente fue el valor del contaminante, y la variable independiente fue una variable indicadora para la intervención, por cada distrito. Los coeficientes de la regresión se interpretan como: intercepto es el valor (promedio del contaminante) pre-intervención, $\beta 1$ es la diferencia post-pre intervención. El coeficiente beta de la regresión lineal evalúa la contaminación aérea antes y después del reordenamiento vehicular, y el valor de $p$ del coeficiente $\beta 1$ determinó la aceptación de la hipótesis.

Para poder comparar, en un modelo de regresión lineal se juntó todas las observaciones de cada contaminante, se incluyó una variable indicadora de cada zona, una variable indicadora del periodo y una interacción entre estas dos variables, obteniéndose tres términos de interacción, uno por cada zona. El valor de p de la interacción entre las variables, determinó si el cambio del contaminante es significativamente diferente entre las Av. Abancay y las otras dos. La Av. Guillermo de la Fuente fue considerada como referencia y se consideró significancia estadística al valor de $p<0,05$.

\section{CONSIDERACIONES ÉTICAS}

Este estudio forma parte del Programa de Investigación «GeoHealth Hub Centered in Peru» de la Universidad Peruana Cayetano Heredia, para lo cual, utilizó los valores de contaminantes ambientales medidos por monitores de DIGESA y está exonerado de aprobación ética.

\section{RESULTADOS}

En la Figura 1 se muestran las concentraciones de contaminantes ambientales en los monitores Ab, SL y AG. Se presentan promedios anuales para el periodo 20072009 y 2013-2015.

Aunque los valores pre-intervención (2007-2009) de $\mathrm{PM}_{2,5}$, $\mathrm{PM}_{10}, \mathrm{NO}_{2}$ y $\mathrm{SO}_{2}$ son más altos en $\mathrm{Ab}$ que en $\mathrm{SL}$ y $\mathrm{AG}$, los valores de $\mathrm{NO}_{2}$ y $\mathrm{SO}_{2}$ estuvieron por debajo del valor de referencia según la ECA Nacional. Sin embargo, si se considera los límites de referencia referidos por la OMS, considerando a $\mathrm{NO}_{2}$ como $40 \mu \mathrm{g} / \mathrm{m}^{3}$ de media anual y $\mathrm{SO}_{2}$ como $20 \mu \mathrm{g} / \mathrm{m}^{3}$ de media en 24 horas, los valores promedios basales de los contaminantes ambientales en Ab para el periodo 2007-2009, superan la directriz recomendada por OMS.

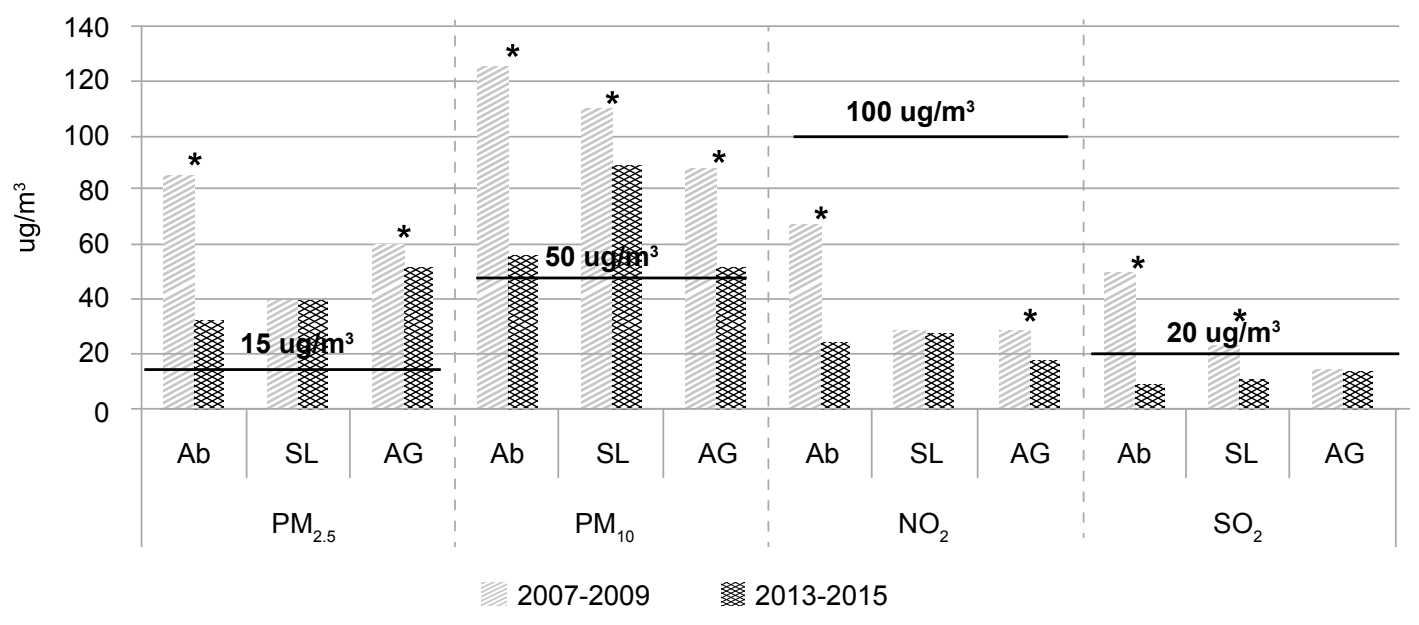

Figura 1. Concentración promedio de los contaminantes del aire en los periodos de estudio pre (2007-2009) y post (2013-2015) ordenamiento vehicular en tres zonas de Lima Metropolitana.

Estándar de Calidad Ambiental (ECA) anual nacional: Material particulado 2,5 $\left(\mathrm{PM}_{2,5}\right)=15 \mu \mathrm{g} / \mathrm{m}^{3} ;$ Material particulado $10(\mathrm{PM} 10)=50 \mu \mathrm{g} / \mathrm{m}^{3}$; Dióxido de nitrógeno $\left(\mathrm{NO}_{2}\right)=100 \mu \mathrm{g} / \mathrm{m}^{3}$; Dióxido de azufre $\left(\mathrm{SO}_{2}\right)=20 \mu \mathrm{g} / \mathrm{m}^{3}$ ( 24 horas).

Ab: Av. Abancay; SL: Av. Guillermo de la Fuente; AG: Av. César Vallejo.

* Valor de $p<0,001$. 
Al evaluar y comparar las concentraciones promedio en cada periodo del estudio se puede observar una reducción significativa en el promedio de concentración de los contaminantes estudiados $(p<0,001)$ en Ab para el periodo 2013-2015, en cambio en SL solo se observó una reducción de los niveles de $\mathrm{PM}_{10}$ y $\mathrm{SO}_{2}(\mathrm{p}<0,001)$.

Asociado a la intervención se observó una reducción del $60 \%$ en la concentración promedio de $\mathrm{PM}_{2,5}$ en $\mathrm{Ab}$ y $35 \%$ en AG. Para $\mathrm{PM}_{10}$, una reducción de $55 \%, 19 \%$ y $40 \%$ fue observado en $\mathrm{Ab}, \mathrm{SL}$ y $\mathrm{AG}$, respectivamente. Para $\mathrm{NO}_{2}$, una reducción de $65 \%, 6 \%$ y $37 \%$ para las zonas de Ab, SL y AG. Finalmente, para $\mathrm{SO}_{2}$, una reducción de $82 \%$ en $\mathrm{Ab}$ versus $50 \%$ en SL (Figura 1).

Se graficó la dispersión (Figura 2) de cada contaminante para observar su distribución en los años. En la Figura 2A se observa una reducción de los niveles de $\mathrm{PM}_{2,5}$ en los años 2013-2015 en Ab, a diferencia de AG mientras que en SL no se visualiza esta disminución. Referente a los demás contaminantes $\mathrm{PM}_{10}, \mathrm{NO}_{2}$ y $\mathrm{SO}_{2}$ también se observa el mismo patrón, una disminución moderada en $\mathrm{Ab}$, a diferencia de las otras dos avenidas (Figura 2B, 2C y 2D).

En la Tabla 1 se muestra los cambios de las concentraciones de los contaminantes. Para $\mathrm{PM}_{2.5}$, se observa una reducción significativa ( $\beta 1$ : $-53,18$; IC 95\%: $-61,49$ a $-44,87$ ) en $\mathrm{Ab}$, una menor reducción en AG ( $\beta 1$ : $-20,75$; IC 95\%: $-27,51$ a -14,00), y ausencia de cambios en SL, por efecto de la intervención (variable indicadora). El coeficiente de regresión obtenido para Ab es significativamente diferente a los de las otras dos avenidas. La misma diferencia se observa para los coeficientes de regresión de $\mathrm{PM}_{10}, \mathrm{SO}_{2}$ y NO $2(p<0,001)$.

Para comparar los cambios de las concentraciones de los contaminantes observados en la Figura 2, a la regresión
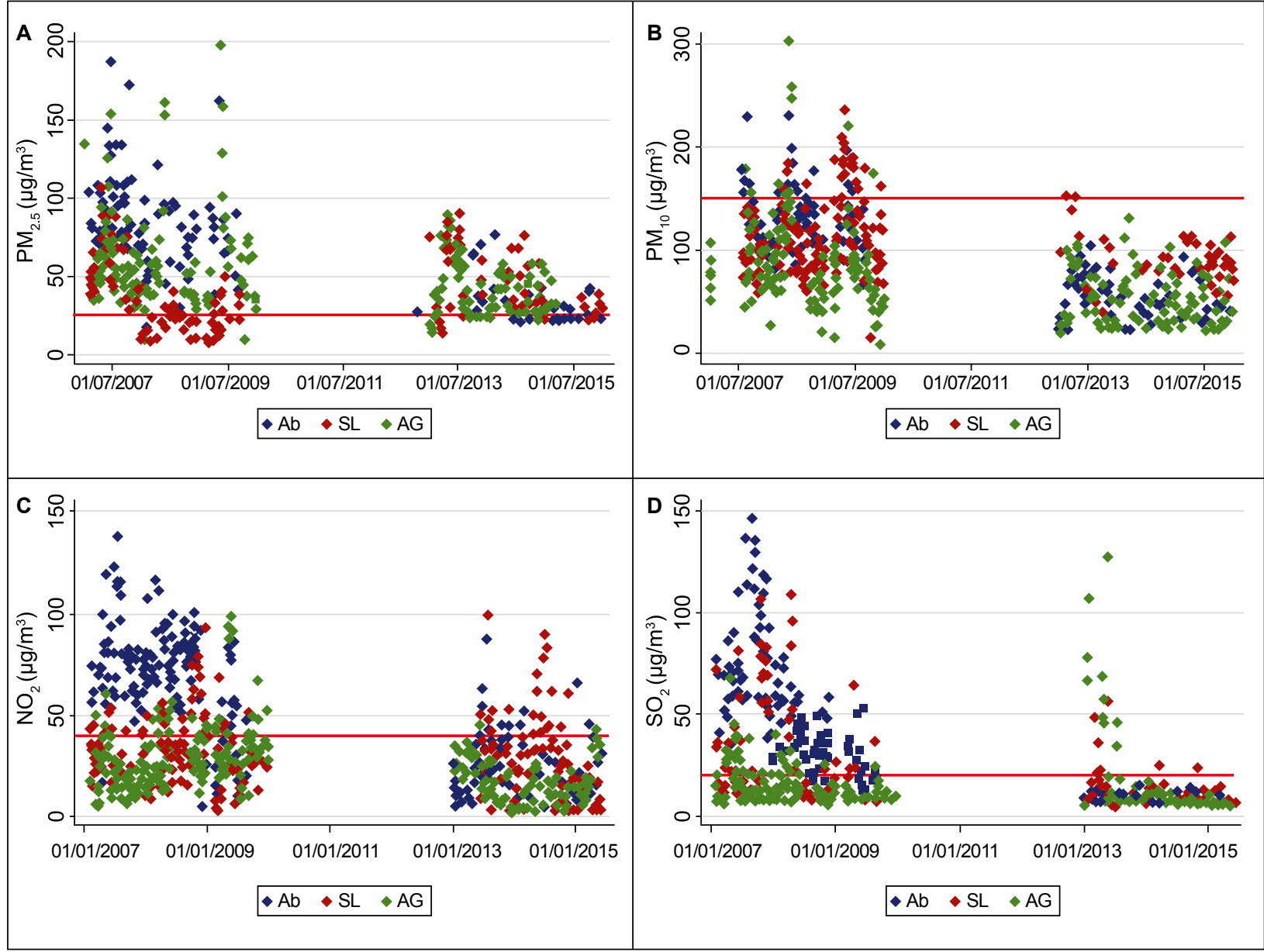

Figura 2. Distribución de los contaminantes ambientales. A. Material particulado 2,5 $\left(\mathrm{PM}_{2,5}\right)$, línea recta en color rojo valor referencial del Estándar de Calidad Ambiental (ECA) promedio 24 horas $\left(\mathrm{PM}_{2,5}=25 \mu \mathrm{g} / \mathrm{m}^{3}\right)$. B. Material particulado $10\left(P M_{10}\right)$, línea recta en color rojo valor referencial ECA promedio 24 horas $\left(\mathrm{PM}_{10}=150 \mu \mathrm{g} / \mathrm{m}^{3}\right)$. C. . Dióxido de nitrógeno $\left(\mathrm{NO}_{2}\right)$, línea recta color rojo valor referencial ECA promedio 24 horas $\left(\mathrm{NO}_{2}=40 \mu \mathrm{g} / \mathrm{m}^{3}\right)$. D. Dióxido de azufre $\left(\mathrm{SO}_{2}\right)$, en línea recta color rojo valor referencial ECA promedio 24 horas $\left(\mathrm{SO}_{2}=20 \mu \mathrm{g} / \mathrm{m}^{3}\right)$.

Ab: Av. Abancay; SL: Av. Guillermo de la Fuente; AG: Av. César Vallejo. 
Tabla 1. Coeficientes de regresión entre el valor de los contaminantes ambientales $\left(\mathrm{PM}_{2,5}, \mathrm{PM}_{10}, \mathrm{SO}_{2}, \mathrm{NO}_{2}\right)$ pre (2007-2009) post (2013-2015) ordenamiento vehicular en tres zonas de Lima Metropolitana.

\begin{tabular}{|c|c|c|c|c|c|c|c|c|}
\hline \multirow{2}{*}{ Zona } & \multicolumn{2}{|l|}{$\mathrm{PM}_{2.5}$} & \multicolumn{2}{|l|}{$\mathrm{PM}_{10}$} & \multicolumn{2}{|l|}{$\mathrm{SO}_{2}$} & \multicolumn{2}{|l|}{$\mathrm{NO}_{2}$} \\
\hline & $\beta 1$ (IC 95\%) & Valor de $p$ & $\beta 1$ (IC 95\%) & Valor de $p$ & $\beta 1$ (IC 95\%) & Valor de $p$ & $\beta 1$ (IC 95\%) & Valor de $p$ \\
\hline$A b$ & $\begin{array}{c}-53,18 \\
(-61,49 a-44,87)\end{array}$ & 0,001 & $\begin{array}{c}-69,01 \\
(-76,50 a-61,53)\end{array}$ & 0,001 & $\begin{array}{c}-41,10 \\
(-47,41 \text { a }-34,80)\end{array}$ & 0,001 & $\begin{array}{c}-43,79 \\
(-49,89 a-37,70)\end{array}$ & 0,001 \\
\hline SL & $\begin{array}{c}-0,06 \\
(-6,53 \text { a } 6,39)\end{array}$ & 0,984 & $\begin{array}{c}-21,01 \\
(-32,77 \text { a }-9,26)\end{array}$ & 0,001 & $\begin{array}{c}-12,51 \\
(-16,99 a-8,03)\end{array}$ & 0,001 & $\begin{array}{c}-2,08 \\
(-6,35 \text { a } 2,17)\end{array}$ & 0,336 \\
\hline AG & $\begin{array}{c}-20,75 \\
(-27,51 \text { a }-14,00)\end{array}$ & 0,001 & $\begin{array}{c}-36,02 \\
(-45,23 a-26,82)\end{array}$ & 0,001 & $\begin{array}{c}-0,63 \\
(-3,72 \text { a } 2,45)\end{array}$ & 0,688 & $\begin{array}{c}-10,45 \\
(-13,54 a-7,36)\end{array}$ & 0,001 \\
\hline
\end{tabular}

$\mathrm{PM}_{25}$ : material particulado 2,5; $\mathrm{PM}_{10}$ : material particulado 10; $\mathrm{SO}_{2}$ : dióxido de azufre; $\mathrm{NO}_{2}$ : dióxido de nitrógeno; $\beta 1$ : coeficiente de regresión (diferencia post-pre ordenamiento vehicular); IC 95\%: intervalo de confianza al 95\%; Ab: Av. Abancay; SL: Av. Guillermo de la Fuente; AG: Av. Cesar Vallejo.

lineal se incorporó un término de interacción generada por las variables periodo y zona, obteniéndose una interacción por cada zona. Los resultados muestran que el cambio observado en la concentración de los contaminantes es diferente entre $\mathrm{Ab}$ para $\mathrm{PM}_{2,5}(\beta 1:-53,11$; IC 95\%: $-63,92$ a -42,30), $\mathrm{PM}_{10}$ ( $\beta 1:-47,99$; IC 95\%: -62,61 a -33,37), $\mathrm{NO}_{2}$ ( $\beta 1:-41,7$; IC 95\%: $-48,18$ a $-35,23), \mathrm{SO}_{2}$ ( $\beta 1$ : $-28,59$; IC 95\%: $-35,23$ a $-21,95$ ) y las otras avenidas (Tabla 2 ).

Finalmente, en 2015 los resultados indican que las concentraciones promedio de PM todavía sobrepasan los estándares permitidos. En ambos casos, las concentraciones de $\mathrm{Ab}\left(\mathrm{PM}_{10}: 51 \pm 22,9 \mu \mathrm{g} / \mathrm{m}^{3} ; \mathrm{PM}_{2,5}: 26 \pm 5,73 \mu \mathrm{g} / \mathrm{m}^{3}\right)$ con el resto. Los valores más altos de $\mathrm{PM}_{10}$ se observan en SL y los de $\mathrm{PM}_{2,5}$ en AG. En relación a $\mathrm{NO}_{2}$ y $\mathrm{SO}_{2}$, las concentraciones promedio son similares en las tres zonas, y están por debajo de los valores referenciales.

\section{DISCUSIÓN}

América Latina está inmersa, como muchas otras regiones, en la problemática de la contaminación del aire como consecuencia del alto índice de urbanización, donde el $72 \%$ de la población reside en centros urbanos ${ }^{(12,22)}$. El Perú se ha convertido en un país altamente urbano y donde el mayor grado de urbanización se encuentra en Lima, considerada una de las ciudades con mayor contaminación del aire exterior ${ }^{(12) . ~ E s t a ~ c o n t a m i n a c i o ́ n ~ t i e n e ~ u n a ~ r e p e r c u s i o ́ n ~}$ principalmente en la salud de niños y adultos ${ }^{(23,24)}$.

El transporte vehicular es el principal responsable de la contaminaciónambiental.Entrelosprincipalescontaminantes emitidos por los vehículos motorizados se encuentran el $\mathrm{CO}, \mathrm{NO}_{2}$ y $\mathrm{PM}_{10}, \mathrm{PM}_{2,5}$. Estudios en megaciudades han demostrado que el $\mathrm{PM}_{2,5}$ y $\mathrm{NO}_{2}$ constituyen los principales

Tabla 2. Coeficientes de regresión que relaciona el valor del contaminante ambiental con términos de interacción periodo-zona.

\begin{tabular}{|c|c|c|c|c|c|c|c|c|}
\hline \multirow{2}{*}{ Variables } & \multicolumn{2}{|l|}{$\mathrm{PM}_{2.5}$} & \multicolumn{2}{|l|}{$\mathrm{PM}_{10}$} & \multicolumn{2}{|l|}{$\mathrm{SO}_{2}$} & \multicolumn{2}{|l|}{$\mathrm{NO}_{2}$} \\
\hline & $\beta 1$ (IC 95\%) & $\begin{array}{l}\text { Valor } \\
\text { de } p\end{array}$ & $\beta 1$ (IC 95\%) & $\begin{array}{l}\text { Valor } \\
\text { de } p\end{array}$ & $\beta 1$ (IC 95\%) & $\begin{array}{l}\text { Valor } \\
\text { de p }\end{array}$ & $\beta 1$ (IC 95\%) & $\begin{array}{l}\text { Valor } \\
\text { de p }\end{array}$ \\
\hline \multicolumn{9}{|l|}{ Periodo } \\
\hline Pre & Ref. & & Ref. & & Ref. & & Ref. & \\
\hline Post & $\begin{array}{c}-0,06 \\
(-7,15 \text { a } 7,01)\end{array}$ & 0,985 & $\begin{array}{c}-21,01 \\
(-32,16 \text { a }-9,87)\end{array}$ & 0,001 & $\begin{array}{c}-12,51 \\
(-16,96 a-8,05)\end{array}$ & 0,001 & $\begin{array}{c}-2,08 \\
(-6,50 \text { a } 2,33)\end{array}$ & 0,354 \\
\hline \multicolumn{9}{|l|}{ Zona } \\
\hline$A b$ & $\begin{array}{c}45,93 \\
(39,35 \text { a } 52,52)\end{array}$ & 0,001 & $\begin{array}{c}15,27 \\
(7,42 \text { a } 23,11)\end{array}$ & 0,001 & $\begin{array}{c}26,86 \\
(22,76 \text { a } 30,95)\end{array}$ & 0,001 & $\begin{array}{c}38,16 \\
(34,38 \text { a 41,93) }\end{array}$ & 0,001 \\
\hline SL & Ref. & & Ref. & & Ref. & & Ref. & \\
\hline$A G$ & $\begin{array}{c}21,55 \\
(15,28 \text { a } 27,82)\end{array}$ & 0,001 & $\begin{array}{c}-22,21 \\
(-29,92 a-14,51)\end{array}$ & 0,001 & $\begin{array}{c}-8,75 \\
(-12,58 a-4,91)\end{array}$ & 0,001 & $\begin{array}{c}-1,04 \\
(-4,64 \text { a } 2,55)\end{array}$ & 0,569 \\
\hline \multicolumn{9}{|l|}{ Periodo-zona } \\
\hline Periodo-Ab & $\begin{array}{c}-53,11 \\
(-63,92 a-42,30)\end{array}$ & 0,001 & $\begin{array}{c}-47,99 \\
(-62,61 \text { a }-33,37)\end{array}$ & 0,001 & $\begin{array}{c}-28,59 \\
(-35,23 a-21,95)\end{array}$ & 0,001 & $\begin{array}{c}-41,71 \\
(-48,18 a-35,23)\end{array}$ & 0,001 \\
\hline Periodo-SL & Ref. & & Ref. & & Ref. & & Ref. & \\
\hline Periodo-AG & $\begin{array}{c}-20,69 \\
(-30,18 a-11,19) \\
\end{array}$ & 0,001 & $\begin{array}{c}-15,00 \\
(-28,91 \mathrm{a}-1,10) \\
\end{array}$ & 0,034 & $\begin{array}{c}11,87 \\
(5,67 \text { a } 18,08)\end{array}$ & 0,001 & $\begin{array}{c}-8,36 \\
(-14,42 a-2,30) \\
\end{array}$ & 0,007 \\
\hline
\end{tabular}

$\mathrm{PM}_{25}$ : material particulado 2,5; $\mathrm{PM}_{10}$ : material particulado 10; $\mathrm{SO}_{2}$ : dióxido de azufre; $\mathrm{NO}_{2}$ : dióxido de nitrógeno; $\beta 1$ : coeficiente de regresión (diferencia post-pre ordenamiento vehicular); IC 95\%: intervalo de confianza al 95\%; Pre: pre-ordenamiento; Post: post-ordenamiento; Ab: Av. Abancay; SL: Av. Guillermo de la Fuente; AG: Av. Cesar Vallejo; Periodo-Ab: interacción entre periodo*Av. Abancay; Periodo-SL: interacción entre periodo*Av. Guillermo de la Fuente; Periodo-AG: interacción entre periodo*Av. César Vallejo; Ref: categoría referencial de la variable cuyo valor es cero. 
contaminantes con riesgos para la salud y se estima que la contaminación del aire exterior se convertirá en la mayor causa ambiental de muerte prematura en las próximas décadas ${ }^{(2)}$.

El impacto de los contaminantes ambientales también estaría relacionado con la producción per cápita del país; así, en China ${ }^{(25)}$, se ha demostrado que la contaminación por $\mathrm{PM}_{2,5}$ causa alteraciones en las respiraciones durante el sueño, lo que ocasionaría un inadecuado descanso conllevando una disminución del rendimiento y producción de los trabajadores, especialmente en industrias ubicadas en avenidas con tránsito elevado.

Como en otras grandes ciudades, Lima no escapa de mostrar una gran variabilidad en los niveles de exposición ambiental ${ }^{(26)}$. El mayor nivel de contaminación en Lima se observa en la zona este y norte, aunque los niveles de $\mathrm{PM}_{2,5}$ ya se encuentran en valores que oscilan entre $20 \mathrm{y}$ $30 \mu \mathrm{g} / \mathrm{m}^{3}$, que son valores más bajos que los observados a inicios del siglo XXI.

Los resultados del presente estudio muestran que las políticas de reordenamiento del tránsito vehicular son importantes en la reducción de los contaminantes ambientales. Sin embargo, aún resultan insuficientes según los valores de $\mathrm{PM}_{2,5}, \mathrm{PM}_{10}$, hallados durante el 2015 en la Av. Abancay, los cuáles continúan por encima del valor referencial nacional. Esto conlleva a plantear que no sólo las fuentes móviles son las que incrementan estos valores. En el caso del $\mathrm{NO}_{2}$ y $\mathrm{SO}_{2}$, se encontró una disminución significativa a valores por debajo del límite de referencia, hecho que podría estar asociado a su disminución de concentración en el diésel y control de fuentes puntuales. El $\mathrm{SO}_{2}$ está asociado a la presencia de industrias en la ciudad y tiene efecto en la salud respiratoria ${ }^{(27)}$. La restricción del tránsito vehicular es un excelente modelo de reducción en corto plazo de $\mathrm{CO}, \mathrm{PM}_{10}, \mathrm{NO}_{2}$ y $_{3}$, tal como, se demuestra en las políticas públicas empleadas por China para reducir la contaminación del aire durante los juegos olímpicos de Beijing en $2008^{(28)}$.

Entre los periodos 2007-2009 y 2013-2015 hay una significativa reducción de los niveles de $\mathrm{PM}_{2,5}, \mathrm{PM}_{10}, \mathrm{NO}_{2}$ y $\mathrm{SO}_{2}$ en Lima Centro, patrón no observado en las zonas sin reordenamiento. Esto demuestra que la aplicación de normativas en zonas de alto tránsito vehicular resulta efectiva. Sin embargo, los niveles de contaminantes como $\mathrm{PM}_{2,5}$ y $\mathrm{PM}_{10}$ no llegan a los valores referenciales recomendados por OMS. Los niveles de $\mathrm{SO}_{2}$ y $\mathrm{NO}_{2}$ llegan a valores por debajo de la referencia de OMS. Finalmente, señalamos que los estándares de calidad de aire con los que se ha comparado este estudio han sido recientemente modificados por el Ministerio del Ambiente (Decreto Supremo $N^{\circ}$ 003-2017-MINAM), por ello, este estudio presenta la línea de base de los efectos de las políticas públicas en la salud humana en megaciudades.
Una de las limitaciones del estudio es el registro discontinuo de los contaminantes ambientales que no permite realizar un análisis de series temporales. El modelo de regresión es una alternativa que permite evaluar cambios en periodos diferentes. Otra de las limitaciones es el cambio en la localización de los aparatos de medición, y la potencial presencia de otras fuentes que disminuyan la contaminación. No obstante, la fortaleza del estudio es que permite evaluar una intervención exitosa en términos de aplicar las medidas analizadas al reducir los niveles de $\mathrm{PM}_{2,5}, \mathrm{PM}_{10}, \mathrm{SO}_{2}, \mathrm{y} \mathrm{NO}_{2}$, a pesar de la falta de acceso libre a datos históricos de contaminantes ambientales, tal como ha sido destacado en una reciente publicación ${ }^{(29)}$. Igualmente, en el tiempo examinado hasta 2015 persisten las intervenciones y en algunas cuadras de la Av. Abancay se observan policías, serenos o agentes del municipio que supervisan se cumplan las normas.

Los resultados del estudio son válidos para la avenida Abancay y no puede generalizarse para toda la ciudad. No obstante, para lograr dicho objetivo se deben medir los niveles de contaminantes atmosféricos en otros puntos representativos del área urbana (por ejemplo, con la metodología utilizada por el Ministerio de Salud y Swisscontact), para confirmar que la disminución en la Av. Abancay no sea sólo una redistribución de los compuestos en otras avenidas o calles. Las medidas de prohibición de giro en vías transitables pueden tener la consecuencia de que los automotores recorran distancias mayores por las calles internas, aumentando de este modo las emisiones en estas vías secundarias.

En conclusión, el reordenamiento vehicular, como medida de gestión municipal, podría estar contribuyendo con la disminución continua de diversos contaminantes, y en un futuro se esperaría, si las intervenciones se extienden, que el impacto hacia los habitantes de Lima Metropolitana sea cada vez menor, mejorando su calidad de vida y estado de salud.

Agradecimientos: A la Dirección General de Salud Ambiental e Inocuidad Alimentaria (DIGESA).

Contribuciones de los autores: La idea fue desarrollada por GFG y VV. Participaron en colección de dato: VT, V y RE. Análisis de datos: VT, LC y KS. Redacción del artículo: VT, CVV y GFG. Revisión del manuscrito final: todos los autores.

Declaración: Las opiniones y afirmaciones contenidas en el artículo son propias de los autores y no reflejan ninguna posición oficial u opinión de ninguna entidad gubernamental.

Fuentes de financiamiento: El presente estudio fue financiado por el Grant U01TW010107 (1/2 Regional GEOHealth hub centered in Peru) de los Institutos Nacionales de Salud de los Estados Unidos (Programa Fogarty).

Conflictos de interés: Los autores declaran no tener conflictos de interés con el presente artículo. 


\section{REFERENCIAS BIBLIOGRÁFICAS}

1. World Health Organization. Ambient air pollution: a global assessment of exposure and burden of disease. 1a ed. Ginebra: WHO; 2016.

2. Wheida A, Nasser A, El Nazer M, Borbon A, Abdel Wahab M, Alfaro SC. Tackling the mortality from long-term exposure to outdoor air pollution in megacities: Lessons from the Greater Cairo case study. Environ Res. 2017;160:223-31.

3. San Miguel R. Calidad del Aire de Lima Metropolitana - Foro de Monitoreo Atmosférico y Taller de Gestión Ambiental del Aire en América Latina. Lima: Municipalidad Metropolitana de Lima; 2006.

4. Thomson I, Bull A. La congestión del tránsito urbano: causas y consecuencias económicas y sociales. Revista de la CEPAL. 2002;76:109-21.

5. Agencia de Cooperación Internacional del Japón (JICA). Encuesta de recolección de información básica del transporte urbano en el área metropolitana de Lima y Callao. Informe Final. Lima: Nippon Koei Co; 2013.

6. Perú, Congreso de la República. Decreto Supremo No. 039-2014-EM: Aprueban Reglamento para la Protección Ambiental en las Actividades de Hidrocarburos. Lima: Congreso de la República; 2014.

7. Mormontoy W, Gastañaga C, Gonzales GF. Blood lead levels among police officers in Lima and Callao, 2004. Int J Hyg Environ Health. 2006;209(6):497-502.

8. Grández B, Marianella K, Gonzáles Portal VR. Contaminación atmosférica por emisión de gases de combustión de los grupos electrógenos de Electro Oriente en el área de influencia: período enero-octubre 2010, en Iquitos-Perú [Tesis de Maestría]. Iquitos: Universidad Nacional de la Amazonía Peruana; 2010.

9. Perú, Congreso de la República. Ley №. 28694: Ley que regula el contenido de azufre en el combustible diesel. Lima: Congreso de la República; 2006.

10. Perú, Congreso de la República. Ley Nº 29237: Ley que crea el Sistema Nacional de Inspecciones Técnicas Vehiculares. Lima: Congreso de la República; 2008.
11. Perú, Ministerio de Transporte y Comunicaciones. Resolución Ministerial $\mathrm{N}^{\circ} 395$ 2012-MTC/02: Cronograma del Régimen Extraordinario de Permanencia para vehículos destinados al servicio de transporte de personas de ámbito provincial de Lima Metropolitana. Lima: MTC, 2013.

12. World Health Organization. Ambient (outdoor) air pollution in cities database 2014 [Internet]. Ginebra: WHO; 2014 [citado el 2 de febrero de 2018]. Disponible en: http://www.who.int/phe/health_topics/outdoorair/databases/cities-2014/en/

13. Perú, Congreso de la República. Ley N²7972: Ley Orgánica de Municipalidades. Lima: Congreso de la República; 2003.

14. Secretaría Técnica del Consejo de Transporte de Lima y Callao. Informe $N^{\circ}$. 005-2011. Implementación de medidas de tránsito en la Av. Abancay: situación actual. Lima: MTC; 2011.

15. Municipalidad Metropolitana de Lima. Plan Regional de Desarrollo Concertado de Lima, 2012-2025 [Internet] Lima: MML; 2012 [citado el 2 de febrero de 2018]. Disponible en: http://www.munlima.gob. pe/images/descargas/gobierno-abierto/ transparencia $/ \mathrm{mml} /$ planeamientoy-organizacion/planeamientoorganizacion/Plan-Desarrollo-LimaMetropolitana-2012-2025.pdf

16. Perú, Corte Suprema. Resolución Suprema No. 768-98-PCM. Comité de Gestión de la Iniciativa del Aire Limpio. Lima: Corte Suprema, 1998

17. CPI. Perú: Población 2017. Market Report [Internet]. Lima: CPI; 2017 [citado el 2 de febrero de 2018]. Disponible en: http://cpi.pe/images/upload/ paginaweb/archivo/26/mr_poblacion_ peru_2017.pdf.

18. Perú, Ministerio de Transporte y Comunicaciones. Secretaría Técnica del Consejo de transporte de Lima y Callao. Perfil de tránsito en las principales vías del área metropolitana de Lima y Callao. Lima: MTC; 2011.

19. Perú, Ministerio del Ambiente. Informe Nacional de la Calidad del Aire 2013-2014. Lima: MINAM; 2016.
20. Perú, Congreso de la República. Decreto Supremo No. 074-2001-PCM: Reglamento de Estándares nacionales de calidad ambiental del aire. Lima: Congreso de la República; 2001.

21. Perú, Congreso de la República. Decreto Supremo No. 003-2008-MINAM. Estándares de calidad ambiental para el aire. Lima: Congreso de la República; 2008.

22. Programa de Naciones Unidas para los Asentamientos Humanos. Estado de las Ciudades de América Latina y el Caribe: Rumbo a una nueva transición urbana, Rio de Janeiro: ONU-Habitat; 2012.

23. Laborde A, Tomasina F, Bianchi F, Bruné MN, Buka I, Comba P, et al. Children's Health in Latin America: The Influence of Environmental Exposures. Environ Health Perspect. 2015;123(3):201-9.

24. Boffetta P. Human cancer from environmental pollutants: the epidemiological evidence. Mutat Res. 2006;608(2):157-62

25. Shen YL, Liu WT, Lee KY, Chuang HC, Chen HW, Chuang KJ. Association of PM 2.5 with sleep-disordered breathing from a population-based study in Northern Taiwan urban areas. Environ Pollut. 2017;233:109-13.

26. Nieuwenhuijsen MJ. Urban and transport planning, environmental exposures and health-new concepts, methods and tools to improve health in cities. Environ Health. 2016;15(Suppl 1):S38.

27. Shima M. Health Effects of Air Pollution: A Historical Review and Present Status. Nihon Eiseigaku Zasshi. 2017;72(3):159-65.

28. Cai H, Xie S. Traffic-related air pollution modeling during the 2008 Beijing Olympic Games: the effects of an oddeven day traffic restriction scheme. Sci Total Environ. 2011;409(10):1935-48.

29. Hernández-Vásquez A, Díaz-Seijas D. Environmental pollution and air pollutants' historical data repositories in Peru. Salud Publica Mex. 2017;59(5):507-508.

Correspondencia: Gustavo F. Gonzales

Dirección: Av. Honorio Delgado 430, Lima 31, Perú Correo electrónico:gustavo.gonzales@upch.pe 